\title{
FAst Sets And Points For Fractional Brownian Motion
}

\author{
BY
}

DAVAR KHOSHNEvisAn* \& ZHAN SHI

University of Utah $\&$ Université Paris VI

\begin{abstract}
Summary. In their classic paper, S. Orey and S.J. Taylor compute the Hausdorff dimension of the set of points at which the law of the iterated logarithm fails for Brownian motion. By introducing "fast sets", we describe a converse to this problem for fractional Brownian motion. Our result is in the form of a limit theorem. From this, we can deduce refinements to the aforementioned dimension result of Orey and Taylor as well as the work of R. Kaufman. This is achieved via establishing relations between stochastic co-dimension of a set and its Hausdorff dimension along the lines suggested by a theorem of S.J. Taylor.
\end{abstract}

Keywords and Phrases. Fast point, fast set, fractional Brownian motion, Hausdorff dimension.

A.M.S. 1991 Subject Classification. 60G15, 60G17, 60J65.

\section{§1. INTRODUCTION}

Suppose $W \triangleq(W(t) ; t \geqslant 0)$ is standard one-dimensional Brownian motion starting at 0 . Continuity properties of the process $W$ form a large part of classical probability theory. In particular, we mention A. Khintchine's law of the iterated logarithm (see, for example, [21, Theorem II.1.9]): for each $t \geqslant 0$, there exists a null set $\mathcal{N}_{1}(t)$ such that for all $\omega \notin \mathcal{N}_{1}(t)$,

$$
\limsup _{h \rightarrow 0^{+}} \frac{|W(t+h)-W(t)|}{\sqrt{h \ln \ln (1 / h)}}=\sqrt{2} .
$$

Later on, P. Lévy showed that $\cup_{t} \geqslant 0 \mathcal{N}_{1}(t)$ is not a null set. Indeed, he showed the existence of a null set $\mathcal{N}_{2}$ outside which

$$
\limsup _{h \rightarrow 0^{+}} \sup _{0 \leqslant r \leqslant 1} \frac{|W(r+h)-W(r)|}{\sqrt{h \ln (1 / h)}}=\sqrt{2} .
$$

\footnotetext{
* Research supported by a grant from the National Security Agency
} 
See [13, p. 168] or [21, Theorem I.2.7], for example. It was observed in [18] that the limsup is actually a limit. Further results in this direction can be found in $[2$, p. 18]. The apparent discrepancy between (1.1) and (1.2) led S. Orey and S.J. Taylor to further study the so-called fast (or rapid) points of $W$. To describe this work, for all $\lambda>0$, define $\mathrm{F}_{1}(\lambda)$ to be the collection of all times $t \geqslant 0$ at which

$$
\limsup _{h \rightarrow 0^{+}} \frac{|W(t+h)-W(t)|}{\sqrt{h \ln (1 / h)}} \geqslant \lambda \sqrt{2} .
$$

The main result of [18] is that with probability one,

$$
\operatorname{dim}\left(\mathrm{F}_{1}(\lambda)\right)=1-\lambda^{2} .
$$

One can think of this as the multi-fractal analysis of white noise. Above and throughout, " $\operatorname{dim}(A)$ " refers to the Hausdorff dimension of $A$. Furthermore, whenever $\operatorname{dim}(A)$ is (strictly) negative, we really mean $A=\varnothing$. Orey and Taylor's discovery of Eq. (1.3) relied on special properties of Brownian motion. In particular, they used the strong Markov property in an essential way. This approach has been refined in $[3,4,11]$, in order to extend (1.3) in several different directions.

Our goal is to provide an alternative proof of Eq. (1.3) which is robust enough to apply to non-Markovian situations. We will do so by (i) viewing $F_{1}(\lambda)$ as a random set and considering its hitting probabilities; and (ii) establishing (within these proofs) links between Eqs. (1.2) and (1.3).

To keep from generalities, we restrict our attention to fractional Brownian motion. With this in mind, let us fix some $\alpha \in] 0,2[$ and define $X \triangleq(X(t) ; t \geqslant 0)$ to be a one-dimensional Gaussian process with stationary increments, mean zero and incremental standard deviation given by,

$$
\|X(t)-X(s)\|_{2}=|t-s|^{\alpha / 2} .
$$

See (1.8) for our notation on $L^{p}(\mathbb{P})$ norms.

The process $X$ is called fractional Brownian motion with index $\alpha-$ hereforth written as $\operatorname{fBM}(\alpha)$. We point out that when $\alpha=1, X$ is Brownian motion.

Let $\overline{\operatorname{dim}}_{M}(E)$ denote the upper Minkowski dimension of a Borel set $E \subset \mathbb{R}^{1}$; see references [17, 24]. Our first result, which is a fractal analogue of Eq. (1.2), is the following limit theorem:

Theorem 1.1. Suppose $X$ is $\operatorname{fBM}(\alpha)$ and $E \subset[0,1]$ is closed. With probability one,

$$
\limsup _{h \rightarrow 0^{+}} \sup _{t \in E} \frac{|X(t+h)-X(t)|}{h^{\alpha / 2} \sqrt{\ln (1 / h)}} \leqslant \sqrt{2 \overline{\operatorname{dim}}_{M}(E)}
$$

On the other hand, with probability one,

$$
\sup _{t \in E} \limsup _{h \rightarrow 0^{+}} \frac{|X(t+h)-X(t)|}{h^{\alpha / 2} \sqrt{\ln (1 / h)}} \geqslant \sqrt{2 \operatorname{dim}(E)} .
$$


Loosely speaking, when $\alpha=1$, Theorem 1.1 is a converse to (1.3). For all $\lambda \geqslant 0$, define $\mathcal{F}_{\alpha}(\lambda)$ to be the collection of all closed sets $E \subset[0,1]$ such that

$$
\limsup _{h \rightarrow 0^{+}} \sup _{t \in E} \frac{|X(t+h)-X(t)|}{h^{\alpha / 2} \sqrt{\ln (1 / h)}} \geqslant \lambda \sqrt{2}
$$

One can think of the elements of $\mathcal{F}_{\alpha}(\lambda)$ as $\lambda$-fast sets. Theorem 1.1 can be recast in the following way.

Corollary 1.2. Suppose $X$ is $\mathrm{fBM}(\alpha)$ and $E \subset[0,1]$ is closed. If $\overline{\operatorname{dim}}_{M}(E)<$ $\lambda^{2}$, then $E \notin \mathcal{F}_{\alpha}(\lambda)$ almost surely. On the other hand, if $\operatorname{dim}(E) \geqslant \lambda^{2}$, then $E \in \mathcal{F}_{\alpha}(\lambda)$.

Remark 1.2.1. An immediate consequence of Theorem 1.1 is the following extension of (1.2):

$$
\limsup _{h \rightarrow 0^{+}} \sup _{0 \leqslant t \leqslant 1} \frac{|X(t+h)-X(t)|}{h^{\alpha / 2} \sqrt{\ln (1 / h)}}=\sqrt{2}, \quad \text { a.s. }
$$

When $\alpha \in] 0,1]$, this is a consequence of [15, Theorem 7]. When $\alpha \in] 1,2[$, the existence of such a modulus of continuity is mentioned in [16, Section 5].

A natural question is: can one replace $E$ by a random set? The first random set that comes to our mind is the zero set. When $\alpha=1$, the process is Brownian motion. Its Markovian structure will be used to demonstrate the following.

Theorem 1.3. Suppose $W$ is Brownian motion. Let $z \triangleq\{s \in[0,1]: W(s)=$ $0\}$. Then, with probability one,

$$
\limsup _{h \rightarrow 0^{+}} \sup _{t \in z} \frac{|W(t+h)|}{\sqrt{h \ln (1 / h)}}=\sup _{t \in z} \limsup _{h \rightarrow 0^{+}} \frac{|W(t+h)|}{\sqrt{h \ln (1 / h)}}=1 \text {. }
$$

Thus, the escape of Brownian motion from zero is slower than Lévy's modulus (1.2).

Next, we come to dimension theorems; see (1.3) for an example. Define the $\lambda$-fast points for $\mathrm{fBM}(\alpha)$ as follows:

$$
\mathrm{F}_{\alpha}(\lambda) \triangleq\left\{t \in[0,1]: \limsup _{h \rightarrow 0^{+}} \frac{|X(t+h)-X(t)|}{h^{\alpha / 2} \sqrt{\ln (1 / h)}} \geqslant \lambda \sqrt{2}\right\} .
$$

In particular, $\mathrm{F}_{1}(\lambda)$ denotes the $\lambda$-fast points of Brownian motion. In [9], R. Kaufman has shown that for any closed $E \subset[0,1]$ and every $\lambda>0$, with probability one,

$$
\operatorname{dim}(E)-\lambda^{2} \leqslant \operatorname{dim}\left(E \cap \mathrm{F}_{1}(\lambda)\right) .
$$


Moreover, there exists a certain fixed closed set $E \subset[0,1]$ for which the above is an equality. Our next aim is to show that the inequality in (1.7) is an equality for many sets $E \subset[0,1]$, and that this holds for all $\mathrm{fBM}(\alpha)$ 's. More precisely, we offer the following:

Theorem 1.4. Suppose $X$ is $\operatorname{fBM}(\alpha), E \subset[0,1]$ is closed and $\lambda>0$. Then, with probability one,

$$
\operatorname{dim}(E)-\lambda^{2} \leqslant \operatorname{dim}\left(E \cap \mathrm{F}_{\alpha}(\lambda)\right) \leqslant \operatorname{dim}_{\mathrm{P}}(E)-\lambda^{2},
$$

where $\operatorname{dim}_{P}$ denotes packing dimension.

See [17] and [24] for definitions and properties of $\operatorname{dim}_{P}$.

In particular, (1.3) holds for the fast points of any fractional Brownian motion. Moreover, when $E=z$ and $\alpha=1$, we have the following dimension analogue of Theorem 1.3. Note that $\mathrm{F}_{1}(\lambda)$ is the set of fast points of $W$ as defined earlier. In other words, it is defined by (1.6) with $X$ replaced by $W$.

Theorem 1.5. Suppose $W$ is Brownian motion and $z \triangleq\{s \in[0,1]: W(s)=$ $0\}$. Then, for all $\lambda>0$, with probability one,

$$
\operatorname{dim}\left(z \cap F_{1}(\lambda)\right)=\frac{1}{2}-\lambda^{2}
$$

A natural question which we have not been able to answer is the following:

Problem 1.5.1. Does Theorem 1.5 have a general analogue for all $\mathrm{f} \operatorname{BM}(\alpha)$ 's?

The proofs of Theorems 1.1, 1.3, 1.4 and 1.5 rely on parabolic capacity techniques and entropy arguments. The entropy methods follow the arguments of [18] closely. On the other hand, the parabolic capacity arguments rely on relationships between the Hausdorff dimension of random sets and stochastic co-dimension (see $\S 2$ ). The latter is a formalization of a particular application of [23, Theorem 4], which can be found in various forms within the proofs of $[1,7,14,19]$. We suspect our formulation has other applications. In $\S 3$, we demonstrate (1.4) while (1.5) and the first inequality (i.e., the lower bound) of Theorem 1.4 are derived in $\S 4$. The proof of the upper bound of Theorem 1.3 appears in $\S 5$; the upper bounds of Theorems 1.4 and 1.5 can be found in $\S 6$ and $\S 7$, respectively; and the lower bounds for Theorems 1.3 and 1.5 are proved simultaneously in $\S 8$.

We conclude the Introduction by mentioning some notation which will be utilized throughout this article. Define the function $\psi$ as

$$
\psi(h) \triangleq \sqrt{2 \ln (1 / h)}, \quad h \in] 0,1[.
$$


By $\bar{\Phi}$ we mean the tail of a standard normal distribution, i.e.

$$
\left.\bar{\Phi}(x) \triangleq \frac{1}{\sqrt{2 \pi}} \int_{x}^{\infty} e^{-u^{2} / 2} d u, \quad x \in\right]-\infty, \infty[
$$

Furthermore, $(\Omega, \mathcal{F}, \mathbb{P})$ denotes our underlying probability space. For any real variable $Z$ on $(\Omega, \mathcal{F}, \mathbb{P})$ and for every $p>0$ we write the $L^{p}(\mathbb{P})$-norm of $Z$ by,

$$
\|Z\|_{p} \triangleq\left(\int_{\Omega}|Z(\omega)|^{p} \mathbb{P}(d \omega)\right)^{1 / p}
$$

Throughout, $X$ denotes $\operatorname{fBM}(\alpha)$ for any $\alpha \in] 0,2[$. However, when we wish to discuss Brownian motion specifically (i.e., when $\alpha=1$ ), we write $W$ instead. In accordance with the notation of Theorem $1.3, z$ will always denote the zero set of $W$ restricted to $[0,1]$. Finally, the collection of all atomless probability measures on a set $E$ is denoted by $\mathcal{P}_{+}(E)$.

Remark. Since the first circulation of this paper, many of the 'gaps' in the inequalities of this paper have been bridged. For instance, in Theorem 1.1, both constants of (1.4) and (1.5) can be computed. This and related material can be found in $[10]$.

Acknowledgements. Much of this work was done while the first author was visiting Université Paris VI. We thank L.S.T.A. and Laboratoire de Probabilités for their generous hospitality. Our warmest thanks are extended to Steve Evans, Mike Marcus, Yuval Peres and Yimin Xiao. They have provided us with countless suggestions, references, corrections and their invaluable counsel. In particular, it was Yuval Peres who showed us the rôle played by packing dimensions as well as allowing us to use his argument (cf. [10]) in the proof of Theorem 2.5.

\section{§2. Preliminaries on Dimension}

In this section, we discuss a useful approach to estimating Hausdorff dimensions of random sets via intersection probabilities.

Let $\mathbf{S}_{0}^{1}$ denote the collection of all Borel measurable subsets of $[0,1]$. We say that $E: \Omega \mapsto \mathbf{S}_{0}^{1}$ is a random set, if $\mathbb{1}_{E}(\omega): \Omega \times \mathbf{S}_{0}^{1} \ni(\omega, E) \mapsto\{0,1\}$ is a random variable in the product measure space. An important class of random

sets are the closed stochastic images $E \triangleq \overline{S[0,1]} \triangleq \overline{\{S(t) ; t \in[0,1]\}}$, where $S$ is a stochastic process with càdlàg sample paths.

Let us begin with some preliminaries on Hausdorff dimension; see $[8,16$, 24] for definitions and further details. Given $s \geqslant 0$ and a Borel set $E \subset[0,1]$, let $\Lambda^{s}(E)$ denote the $s$-dimensional Hausdorff measure of $E$. Recall that the Hausdorff dimension - $\operatorname{dim}(E)-$ of $E$ is defined by: $\operatorname{dim}(E) \triangleq \inf \{s>0$ : $\left.\Lambda^{s}(E)<\infty\right\}$. When it is finite, $\Lambda^{s}(E)$ extends nicely to a Carathéodory outer 
measure on analytic subsets of $[0,1]$. By a slight abuse of notation, we continue to denote this outer measure by $\Lambda^{s}$.

Suppose $E$ is a random set. Since we can economically cover $E$ with intervals with rational endpoints, $\operatorname{dim}(E)$ is a random variable. We will use this without further mention.

Typically, computing upper bounds for $\operatorname{dim}(E)$ is not very difficult: find an economical cover $\left(I_{j}\right)$ of $E$, whose diameter is $h$ or less and compute $\sum_{j}\left|I_{j}\right|^{s}$. Obtaining good lower bounds for $\operatorname{dim}(E)$ is the harder of the two bounds. The standard method for doing this is to utilize the connections between Hausdorff dimension and potential theory. For any $\mu \in \mathcal{P}_{+}(E)$ and all $\beta>0$, define,

$$
A_{\beta}(\mu) \triangleq \sup _{0<h \leqslant 1 / 2} \sup _{t \in[h, 1-h]} \frac{\mu[t-h, t+h]}{h^{\beta}} .
$$

(It is possible that $A_{\beta}(\mu)=\infty$.) We need the following connection between Hausdorff dimension and potential theory; while it is only half of Frostman's lemma of classical potential theory, we refer to it as 'Frostman's lemma' for brevity.

Lemma 2.1. (Frostman's Lemma; [8, p. 130]) Suppose $E \in \mathbf{S}_{0}^{1}$ satisfies $\beta<\operatorname{dim}(E)$. Then there exists $\mu \in \mathcal{P}_{+}(E)$ for which $A_{\beta}(\mu)<\infty$.

Thus, a method for obtaining lower bounds on $\operatorname{dim}(E)$ is this: find a probability measure $\mu$ which lives on $E$ and show that $A_{\beta}(\mu)<\infty$. If this can be done for some $\beta>0$, then $\operatorname{dim}(E) \geqslant \beta$. In general, this is all which can be said. However, if the set $E$ in question is a random set in the sense of the first paragraph of this section, there is an abstract version of [23, Theorem 4] which can be used to bound $\operatorname{dim}(E)$ from below; see also [1]. We shall develop this next. Define the upper stochastic co-dimension $(\overline{\mathrm{co}-\mathrm{dim}})$ of a random set $E$ by

$\overline{\operatorname{co}-\operatorname{dim}}(E) \triangleq \inf \left\{\beta \in[0,1]: \forall G \in \mathbf{S}_{0}^{1}\right.$ with $\left.\operatorname{dim}(G)>\beta, \mathbb{P}(E \cap G \neq \varnothing)=1\right\}$.

In order to make our definition sensible and complete, we need to define inf $\varnothing \triangleq$ 1.

Remark 2.1.1. In applications, we often need the following fact: if $G \in \mathbf{S}_{0}^{1}$ satisfies $\operatorname{dim}(G)>\overline{\operatorname{co}-\operatorname{dim}}(E)$, then $\mathbb{P}(E \cap G \neq \varnothing)=1$.

In this section we present two results about stochastic co-dimension, the first of which is the following.

Theorem 2.2. Suppose $E$ is a random set. Then, for all $G \in \mathbf{S}_{0}^{1}$,

$$
\operatorname{dim}(E \cap G) \geqslant \operatorname{dim}(G)-\overline{\operatorname{co}-\operatorname{dim}}(E), \quad \text { a.s. }
$$

As mentioned earlier, Theorem 2.2 is an abstract form of a part of [23, Theorem 4]. This kind of result has been implicitly used in several works. For 
example, see $[7,14,19,20]$. To prove it, let us introduce an independent symmetric stable Lévy process $S_{\gamma} \triangleq\left(S_{\gamma}(t) ; t \geqslant 0\right)$ of index $\left.\gamma \in\right] 0,1[$. The following two facts are due to J. Hawkes; cf. [6].

Lemma 2.3. Suppose $G \in \mathbf{S}_{0}^{1}$ satisfies $\operatorname{dim}(G)<1-\gamma$. Then, with probability one, $\overline{S_{\gamma}[0,1]} \cap G=\varnothing$.

Lemma 2.4. Suppose $G \in \mathbf{S}_{0}^{1}$ satisfies $\operatorname{dim}(G)>1-\gamma$. Then, $\mathbb{P}\left(\overline{S_{\gamma}[0,1]} \cap G \neq\right.$ $\varnothing)>0$. Furthermore, on $\left(\overline{S_{\gamma}[0,1]} \cap G \neq \varnothing\right)$,

$$
\operatorname{dim}\left(\overline{S_{\gamma}[0,1]} \cap G\right)=\operatorname{dim}(G)+\gamma-1, \quad \text { a.s. }
$$

Historically, the above results are stated with $\overline{S_{\gamma}[0,1]}$ replaced by $S_{\gamma}[0,1]$. By symmetry, semi-polar sets are polar for $S_{\gamma}$. Therefore, the same facts hold for $\overline{S_{\gamma}[0,1]}$.

We can now proceed with Theorem 2.2.

Proof of Theorem 2.2. Without loss of generality, we can assume that the compact set $G$ satisfies $\operatorname{dim}(G)>\overline{\operatorname{co}-\operatorname{dim}}(E)$. With this reduction in mind, let us choose a number $\gamma \in] 0,1[$ satisfying,

$$
\gamma>1-\operatorname{dim}(G)+\overline{\operatorname{co}-\operatorname{dim}}(E) .
$$

Choose the process $S_{\gamma}$ as in the earlier part of this section. Since $\gamma>1-\operatorname{dim}(G)$, it follows from Lemma 2.4 that $\kappa \triangleq \mathbb{P}\left(\overline{S_{\gamma}[0,1]} \cap G \neq \varnothing\right)>0$. By (2.3),

$$
\begin{aligned}
\kappa & =\mathbb{P}\left(\overline{S_{\gamma}[0,1]} \cap G \neq \varnothing, \operatorname{dim}\left(\overline{S_{\gamma}[0,1]} \cap G\right)=\operatorname{dim}(G)+\gamma-1\right) \\
& \leqslant \mathbb{P}\left(\overline{S_{\gamma}[0,1]} \cap G \neq \varnothing, \operatorname{dim}\left(\overline{S_{\gamma}[0,1]} \cap G\right)>\overline{\operatorname{co}-\operatorname{dim}(E)),}\right.
\end{aligned}
$$

where we have used (2.4) in the last inequality. In view of Remark 2.1.1, $\kappa$ is bounded above by $\mathbb{P}\left(\overline{S_{\gamma}[0,1]} \cap G \cap E \neq \varnothing\right)$. Applying Lemma 2.3 gives

$$
\begin{aligned}
\kappa & \leqslant \mathbb{P}\left(\overline{S_{\gamma}[0,1]} \cap G \cap E \neq \varnothing, \operatorname{dim}(G \cap E) \geqslant 1-\gamma\right) \\
& \leqslant \mathbb{P}\left(\overline{S_{\gamma}[0,1]} \cap G \neq \varnothing, \operatorname{dim}(G \cap E) \geqslant 1-\gamma\right) \\
& =\kappa \mathbb{P}(\operatorname{dim}(G \cap E) \geqslant 1-\gamma) .
\end{aligned}
$$

The last line utilizes the independence of $S_{\gamma}$ and $E$. Since $\kappa>0$, it follows that for all $\gamma$ satisfying (2.4), $\operatorname{dim}(G \cap E) \geqslant 1-\gamma$, almost surely. Let $\gamma \downarrow 1-\operatorname{dim}(G)+$ $\overline{\operatorname{co}-\operatorname{dim}}(E)$ along a rational sequence to obtain the result.

Next, we present the second result of this Section. It is an immediate consequence of the estimates of [10, Section 3] and Theorem 2.2 above. 
Theorem 2.5. Suppose $\left(E_{n} ; n \geqslant 1\right)$ is a countable collection of open random

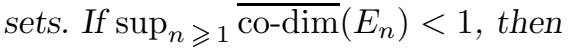

$$
\overline{\operatorname{co}-\operatorname{dim}}\left(\bigcap_{n=1}^{\infty} E_{n}\right)=\sup _{n \geqslant 1} \overline{\operatorname{co-dim}}\left(E_{n}\right) .
$$

In particular, $\mathbb{P}\left(\cap_{n} \geqslant 1 E_{n} \neq \varnothing\right)=1$.

Informally speaking, this is a dual to the fact that for all $F_{n} \in \mathbf{S}_{0}^{1}(n=$ $1,2, \ldots), \operatorname{dim}\left(\cup_{n=1}^{\infty} F_{n}\right)=\sup _{n} \geqslant 0 \operatorname{dim}\left(F_{n}\right)$.

\section{§3. TheOrem 1.1: UpPer Bound}

Define the set of "near-fast points" as follows: for all $\lambda, h>0$,

$$
\mathrm{F}_{\alpha}(\lambda, h) \triangleq\left\{t \in[0,1]: \sup _{t \leqslant s \leqslant t+h}|X(s)-X(t)| \geqslant \lambda h^{\alpha / 2} \psi(h)\right\} .
$$

Next, for any $R, \eta>1$, all integers $j \geqslant 1$ and every integer $0 \leqslant m<R^{\eta j}+1$, define

$$
I_{m, j}^{\eta} \triangleq\left[m R^{-\eta j},(m+1) R^{-\eta j}\right] .
$$

Finally, define for the above parameters,

$$
P_{m, j}^{\lambda, \alpha} \triangleq \mathbb{P}\left(I_{m, j}^{\eta} \cap \mathrm{F}_{\alpha}\left(\lambda, R^{-j}\right) \neq \varnothing\right) .
$$

The main technical estimate which we shall need in this section is the following:

Lemma 3.1. Let $X$ be $\mathrm{fBM}(\alpha)$, where $\alpha \in] 0,2[$. For all $\lambda>0, \varepsilon \in] 0,1[, \eta>1$ and all $R>1$, there exists $J_{1}=J_{1}(\varepsilon, \alpha, \eta, \lambda, R) \in\left[2, \infty\left[\right.\right.$ such that for all $j \geqslant J_{1}$ and all $m \geqslant 0$,

$$
P_{m, j}^{\lambda, \alpha} \leqslant R^{-\lambda^{2}(1-\varepsilon) j} .
$$

Remark 3.1.1. Part of the assertion is that $J_{1}$ does not depend on the choice of $m$.

Proof. By stationarity and scaling,

$$
P_{m, j}^{\lambda, \alpha}=\mathbb{P}\left(\sup _{\left.0 \leqslant t \leqslant R^{-(1-\eta) j}\right)} \sup _{0 \leqslant s \leqslant 1}|X(s+t)-X(s)| \geqslant \lambda \psi\left(R^{-j}\right)\right) .
$$

We obtain the lemma by applying standard estimates and [12, Lemma 3.1] to the Gaussian process $(X(s+t)-X(t) ; s, t \geqslant 0)$.

The proof of the upper bound is close to its counterpart [18]; cf. the first part of Theorem 2 therein. 
Proof of Theorem 1.1: upper bound. Recall (3.1) and (3.2). Consider a fixed closed set $E \subset[0,1]$. Fix $R, \eta>1$ and $\lambda>0$. Define for all integers $m \geqslant 0$,

$$
J_{k} \triangleq \sum_{j \geqslant k} \sum_{m \geqslant 0} \mathbb{1}_{\left\{I_{m, j}^{\eta} \cap \mathrm{F}_{\alpha}\left(\lambda, R^{-j}\right) \neq \varnothing\right\}} \mathbb{1}_{\left\{I_{m, j}^{\eta} \cap E \neq \varnothing\right\}} .
$$

By (3.3) and Lemma 3.1, for all $\varepsilon \in] 0,1\left[\right.$, there exists $J_{1}=J_{1}(\varepsilon, \alpha, \eta, \lambda, R) \in$ $\left[2, \infty\left[\right.\right.$ such that for all $k \geqslant J_{1}$,

$$
\begin{aligned}
\left\|\partial_{k}\right\|_{1} & =\sum_{j \geqslant k} \sum_{m \geqslant 0} P_{m, j}^{\lambda, \alpha} \mathbb{1}_{\left\{I_{m, j}^{\eta} \cap E \neq \varnothing\right\}} \leqslant \sum_{j \geqslant k} \sum_{m \geqslant 0} R^{-\lambda^{2}(1-\varepsilon) j} \mathbb{1}_{\left\{I_{m, j}^{\eta} \cap E \neq \varnothing\right\}} \\
& \leqslant \sum_{j \geqslant k} R^{-\lambda^{2}(1-\varepsilon) j} M\left(R^{-\eta j} ; E\right),
\end{aligned}
$$

where $M(\varepsilon ; E)$ denotes the $\varepsilon-$ capacity of $E$. That is, it is the maximal number of points in $E$ which are at least $\varepsilon$ apart; see [5]. On the other hand, by definition, for all $\delta \in] 0,1\left[\right.$, there exists $J_{2}=J_{2}(\delta, R, \eta) \in\left[2, \infty\left[\right.\right.$, such that for all $j \geqslant J_{2}$, $M\left(R^{-\eta j} ; E\right) \leqslant R^{\eta j(1+\delta) \overline{\operatorname{dim}}_{M}(E)}$. Hence, for all $k \geqslant J_{1} \vee J_{2}$,

$$
\left\|\mathcal{J}_{k}\right\|_{1} \leqslant \sum_{j \geqslant k} R^{-j\left(\lambda^{2}(1-\varepsilon)-\eta(1+\delta) \overline{\operatorname{dim}}_{M}(E)\right)} .
$$

It may help to recall that $J_{1} \vee J_{2}$ depends only on the parameters $(\lambda, \alpha, R, \eta, \delta, \varepsilon)$. Let us pick these parameters so that $\lambda^{2}(1-\varepsilon)>\eta(1+\delta) \overline{\operatorname{dim}}_{M}(E)$. It is easy to see that for this choice of parameters, $\sum_{k}\left\|\mathcal{J}_{k}\right\|_{1}<\infty$. By the Borel-Cantelli lemma, with probability one, there exists a finite random variable $k_{0}$ such that for all $k \geqslant k_{0}, \partial_{k}=0$. In other words, with probability one, for all $j \geqslant k_{0}$, $\mathrm{F}_{\alpha}\left(\lambda, R^{-j}\right) \cap E=\varnothing$. Rewriting the above, we see that with probability one, for all $j \geqslant k_{0}, \sup _{t \in E} \sup _{t \leqslant s \leqslant t+R^{-j}}|X(s)-X(t)| \leqslant \lambda R^{-j \alpha / 2} \psi\left(R^{-j}\right)$. Take any $h \leqslant R^{-k_{0}}$. There exists a $j \geqslant k_{0}$, so that $R^{-j-1} \leqslant h \leqslant R^{-j}$. It follows that for all $h \leqslant R^{-k_{0}}$

$$
\begin{aligned}
\sup _{t \in E}|X(t+h)-X(t)| & \leqslant \sup _{t \in E} \sup _{t \leqslant s \leqslant t+R^{-j}}|X(s)-X(t)| \\
& \leqslant \lambda R^{-j \alpha / 2} \psi\left(R^{-j}\right) \leqslant \lambda R^{\alpha / 2} h^{\alpha / 2} \psi(h),
\end{aligned}
$$

since $\psi$ is monotone decreasing. This implies that whenever $\lambda^{2}(1-\varepsilon)>\eta(1+$ $\delta) \overline{\operatorname{dim}}_{M}(E)$,

$$
\limsup _{h \rightarrow 0^{+}} \sup _{t \in E} \frac{|X(t+h)-X(t)|}{h^{\alpha / 2} \psi(h)} \leqslant \lambda R^{\alpha / 2}, \quad \text { a.s. }
$$

Along rational sequences, let $\varepsilon, \delta \rightarrow 0^{+}, \eta, R \rightarrow 1^{+}$and $\lambda^{2} \downarrow \overline{\operatorname{dim}}_{M}(E)-$ in this order - to see that with probability one,

$$
\limsup _{h \rightarrow 0^{+}} \sup _{t \in E} \frac{|X(t+h)-X(t)|}{h^{\alpha / 2} \psi(h)} \leqslant \sqrt{\overline{\operatorname{dim}}_{M}(E)} .
$$




\section{§4. TheOrEms 1.1 AND 1.4: LOWER Bounds}

For each closed set $E \subset[0,1]$ and for every $\mu \in \mathcal{P}_{+}(E)$ and $h, \lambda>0$, define,

$$
I_{\mu}(h ; \lambda) \triangleq \int_{0}^{1} \mu(d t) \mathbb{1}_{\left\{X(t+h)-X(t)>\lambda h^{\alpha / 2} \psi(h)\right\}}
$$

The key technical estimate of this section is the following:

Lemma 4.1. Suppose $E \subset[0,1]$ is compact, $\mu \in \mathcal{P}_{+}(E)$. For any $\left.\varepsilon \in\right] 0,1[$ and $\lambda>0$, there exists a small $\left.h_{0} \in\right] 0,1[$ such that for all $h \in] 0, h_{0}[$,

$$
\frac{\left\|I_{\mu}(h ; \lambda)\right\|_{2}^{2}}{\left\|I_{\mu}(h ; \lambda)\right\|_{1}^{2}} \leqslant 1+\varepsilon+4 \sup _{h^{1-\varepsilon} \leqslant t \leqslant 1-h^{1-\varepsilon}} \frac{\mu\left(\left[t-h^{1-\varepsilon}, t+h^{1+\varepsilon}\right]\right)}{\bar{\Phi}(\lambda \psi(h))} .
$$

Proof. Since $X$ has stationary increments,

$$
\left\|I_{\mu}(h ; \lambda)\right\|_{1}=\bar{\Phi}(\lambda \psi(h)) .
$$

We proceed with the estimate for the second moment. Define,

$$
\begin{aligned}
a & \triangleq \lambda \psi(h) \\
U & \triangleq \frac{X(s+h)-X(s)}{h^{\alpha / 2}} \\
V & \triangleq \frac{X(t+h)-X(t)}{h^{\alpha / 2}} \\
\rho & \triangleq\|U V\|_{1} .
\end{aligned}
$$

Then, ignoring the dependence on $(h, s, t)$,

$$
\left\|I_{\mu}(h ; \lambda)\right\|_{2}^{2}=Q_{1}+Q_{2}+Q_{3}
$$

where,

$$
\begin{aligned}
& Q_{1} \triangleq \int_{0}^{1} \mu(d t) \int_{0}^{1} \mu(d s) \mathbb{1}_{\left\{\rho \leqslant(\ln (1 / h))^{-2}\right\}} \mathbb{P}(U \geqslant a, V \geqslant a), \\
& Q_{2} \triangleq 2 \int_{0}^{1} \mu(d t) \int_{0}^{t} \mu(d s) \mathbb{1}_{\left\{\rho>(\ln (1 / h))^{-2}\right\}} \mathbb{1}_{\{t-s>2 h\}} \mathbb{P}(U \geqslant a, V \geqslant a), \\
& Q_{3} \triangleq 2 \int_{0}^{1} \mu(d t) \int_{0}^{t} \mu(d s) \mathbb{1}_{\left\{\rho>(\ln (1 / h))^{-2}\right\}} \mathbb{1}_{\{t-s \leqslant 2 h\}} \mathbb{P}(U \geqslant a, V \geqslant a) .
\end{aligned}
$$


We estimate each term separately. The critical term is $Q_{1}$. Write $x^{+}=\max (x, 0)$ for any $x$. If $\rho<1 / 4$,

$$
\begin{aligned}
\mathbb{P}(U \geqslant a, V \geqslant a) & =\frac{1}{2 \pi \sqrt{1-\rho^{2}}} \int_{a}^{\infty} \int_{a}^{\infty} \exp \left(-\frac{x^{2}+y^{2}-2 \rho x y}{2\left(1-\rho^{2}\right)}\right) d x d y \\
& \leqslant \frac{1}{2 \pi \sqrt{1-\rho^{2}}} \int_{a}^{\infty} \int_{a}^{\infty} \exp \left(-\frac{\left(1-4 \rho^{+}\right)\left(x^{2}+y^{2}\right)}{2\left(1-\rho^{2}\right)}\right) d x d y \\
& =\frac{\sqrt{1-\rho^{2}}}{1-4 \rho^{+}}\left(\bar{\Phi}\left(a \sqrt{\frac{1-4 \rho^{+}}{1-\rho^{2}}}\right)\right)^{2} .
\end{aligned}
$$

According to Mill's ratio for Gaussian tails ([22, p. 850]), for any $x>1$,

$$
\frac{1-x^{-2}}{\sqrt{2 \pi} x} \exp \left(-\frac{x^{2}}{2}\right) \leqslant \bar{\Phi}(x) \leqslant \frac{1}{\sqrt{2 \pi} x} \exp \left(-\frac{x^{2}}{2}\right) .
$$

Therefore, using the fact that $\mu$ is an atomless probability measure, we have,

$$
\begin{aligned}
& Q_{1} \leqslant \sup _{r:-1<r \leqslant(\ln (1 / h))^{-2}} \frac{\sqrt{1-r^{2}}}{1-4 r^{+}}\left(\Phi\left(\lambda \psi(h) \sqrt{\frac{1-4 r^{+}}{1-r^{2}}}\right)\right)^{2} \\
& \leqslant[\bar{\Phi}(\lambda \psi(h))]^{2} \sup _{r:-1<r \leqslant(\ln (1 / h))^{-2}}\left(1-\frac{1}{(\lambda \psi(h))^{2}}\right)^{-2} \frac{\left(1-r^{2}\right)^{3 / 2}}{\left(1-4 r^{+}\right)^{2}} \times \\
& \quad \times \exp \left(\frac{(\lambda \psi(h))^{2}\left(4 r^{+}-r^{2}\right)}{1-r^{2}}\right) .
\end{aligned}
$$

Since $(\psi(h))^{2}(\ln (1 / h))^{-2}=o(1)$ (as $h$ goes to 0$)$, this leads to:

$$
Q_{1} \leqslant B(h ; \lambda)[\bar{\Phi}(\lambda \psi(h))]^{2},
$$

where $B(h ; \lambda)$ is such that, for any $\lambda>0$,

$$
\lim _{h \rightarrow 0^{+}} B(h ; \lambda)=1 .
$$

To estimate $Q_{3}$, use the trivial inequality $\mathbb{P}(U \geqslant a, V \geqslant a) \leqslant \mathbb{P}(U \geqslant a)$, to see that

$$
\begin{aligned}
Q_{3} & \leqslant 2 \sup _{2 h \leqslant t \leqslant 1} \mu([t-2 h, t]) \bar{\Phi}(\lambda \psi(h)) \\
& \leqslant 2 \sup _{2 h \leqslant t \leqslant 1-2 h} \mu([t-2 h, t+2 h]) \bar{\Phi}(\lambda \psi(h)) .
\end{aligned}
$$

Finally, we need to approximate $Q_{2}$. Directly computing, note that when $s<$ $t-2 h$,

$$
\begin{aligned}
\rho & =\frac{|t-s-h|^{\alpha}+|t-s+h|^{\alpha}-2|t-s|^{\alpha}}{2 h^{\alpha}} \\
& =\frac{1}{2}\left(\frac{t-s}{h}\right)^{\alpha}\left[\left(1-\frac{h}{t-s}\right)^{\alpha}+\left(1+\frac{h}{t-s}\right)^{\alpha}-2\right] .
\end{aligned}
$$


By Taylor expansion of $(1 \pm x)^{\alpha}$, we see that for all $|x| \leqslant \frac{1}{2}$,

$$
(1-x)^{\alpha}+(1+x)^{\alpha}-2=\frac{\alpha(\alpha-1)}{2}\left[\left(1-\xi_{1}\right)^{\alpha-2}+\left(1-\xi_{2}\right)^{\alpha-2}\right] x^{2},
$$

where $\left|\xi_{i}\right| \leqslant \frac{1}{2}$ for $i=1,2$. In particular, for all $|x| \leqslant \frac{1}{2}$,

$$
\left|(1-x)^{\alpha}+(1+x)^{\alpha}-2\right| \leqslant 2^{3-\alpha} x^{2} .
$$

In other words, when $s<t-2 h, \rho \leqslant\{2 h /(t-s)\}^{2-\alpha}$. On the other hand, if we also know that $\rho>(\ln (1 / h))^{-2}$, it follows that for all $h$ small, $|t-s| \leqslant 2 h(\ln (1 / h))^{2 /(2-\alpha)} \leqslant h^{1-\varepsilon}$. Since $\mathbb{P}(U \geqslant a, V \geqslant a) \leqslant \bar{\Phi}(\lambda \psi(h))$, we obtain the following: for all $\varepsilon>0$, there exists $\left.h_{1} \in\right] 0,1$ [ such that for all $\left.h \in\right] 0, h_{1}[$,

$$
Q_{2} \leqslant 2 \sup _{h^{1-\varepsilon} \leqslant t \leqslant 1-h^{1-\varepsilon}} \mu\left(\left[t-h^{1-\varepsilon}, t+h^{1-\varepsilon}\right]\right) \bar{\Phi}(\lambda \psi(h)) .
$$

Together with (4.7), we obtain: for all $\varepsilon>0$, there exists $\left.h_{2} \in\right] 0,1[$ such that for all $h \in] 0, h_{2}[$,

$$
Q_{2}+Q_{3} \leqslant 4 \sup _{h^{1-\varepsilon} \leqslant t \leqslant 1-h^{1-\varepsilon}} \mu\left(\left[t-h^{1-\varepsilon}, t+h^{1-\varepsilon}\right]\right) \bar{\Phi}(\lambda \psi(h)) .
$$

Combining this with (4.2)-(4.3) and (4.5)-(4.7), we obtain the result.

Now we can prove the lower bounds in Theorems 1.1 and 1.4.

Proof of Theorems 1.1 and 1.4: lower bounds. By Frostman's lemma (Lemma 2.1), for any $\beta<\operatorname{dim}(E)$, there exists a $\mu \in \mathcal{P}_{+}(E)$, such that for all $h \in] 0,1[$ small,

$$
\sup _{h \leqslant t \leqslant 1-h} \mu([t-h, t+h]) \leqslant h^{\beta} .
$$

For such a $\mu$, use Lemma 4.1 to see that for all $\varepsilon \in] 0,1[$ and $\lambda>0$, there exists $\left.h_{3} \in\right] 0,1[$ such that whenever $h \in] 0, h_{3}[$,

$$
\frac{\left\|I_{\mu}(h ; \lambda)\right\|_{2}^{2}}{\left\|I_{\mu}(h ; \lambda)\right\|_{1}^{2}} \leqslant 1+\varepsilon+\frac{4 h^{(1-\varepsilon) \beta}}{\bar{\Phi}(\lambda \psi(h))} .
$$

According to Mill's ratio for Gaussian tails (see (4.4)), for any $\lambda>0$, there exists a small $h_{4}>0$, such that for all $\left.h \in\right] 0, h_{4}\left[, \bar{\Phi}(\lambda \psi(h)) \geqslant h^{\lambda^{2}} / 4 \lambda \sqrt{\ln (1 / h)}\right.$. Hence, for all $h \in] 0, h_{3} \wedge h_{4}[$,

$$
\frac{\left\|I_{\mu}(h ; \lambda)\right\|_{2}^{2}}{\left\|I_{\mu}(h ; \lambda)\right\|_{1}^{2}} \leqslant 1+\varepsilon+16 \lambda h^{(1-\varepsilon) \beta-\lambda^{2}} \sqrt{\ln (1 / h)}
$$


By choosing $\lambda$ such that $(1-\varepsilon) \beta>\lambda^{2}$ we can deduce that for all $\left.h \in\right] 0, h_{3} \wedge h_{4}[$, $\left\|I_{\mu}(h ; \lambda)\right\|_{2}^{2}\left\|I_{\mu}(h ; \lambda)\right\|_{1}^{-2} \leqslant 1+2 \varepsilon$. Applying the Paley-Zygmund inequality $([8$, p. 8$]$ ), we see that

$$
\mathbb{P}\left(I_{\mu}(h ; \lambda)>0\right) \geqslant \frac{1}{1+2 \varepsilon} .
$$

We are ready to complete the proof. Define,

$$
\mathcal{A}_{\alpha}(\lambda, h) \triangleq\left\{t \in[0,1]: \sup _{0 \leqslant r \leqslant h} \frac{|X(t+r)-X(t)|}{r^{\alpha / 2} \psi(r)}>\lambda\right\} .
$$

This is the collection of all $h$-approximate $\lambda$-fast points. Observe that for each $h>0, \mathcal{A}_{\alpha}(\lambda, h)$ is an open subset of $[0,1]$. If $I_{\mu}(h ; \lambda)>0\left(\mu \in \mathcal{P}_{+}(E)\right)$, then $E \cap \mathcal{A}_{\alpha}(\lambda, h) \neq \varnothing$. By (4.8), we see that as long as $(1-\varepsilon) \beta>\lambda^{2}$, then for all $h \in] 0, h_{3} \wedge h_{4}\left[, \mathbb{P}\left(E \cap \mathcal{A}_{\alpha}(\lambda, h) \neq \varnothing\right) \geqslant(1+2 \varepsilon)^{-1}\right.$. Note that if $h \leqslant h^{\prime}$, then $\mathcal{A}_{\alpha}(\lambda, h) \subset \mathcal{A}_{\alpha}\left(\lambda, h^{\prime}\right)$. Hence, for all $\lambda^{2} \in\left[0, \operatorname{dim}(E)\left[, \mathbb{P}\left(\mathcal{A}_{\alpha}(\lambda, h) \cap E \neq \varnothing, \forall h>\right.\right.\right.$ $0)=1$. By Theorem 2.5, for all $\lambda^{2} \in\left[0, \operatorname{dim}(E)\left[, \mathbb{P}\left(\cap_{h>0} \mathcal{A}_{\alpha}(\lambda, h) \cap E \neq \varnothing\right)=1\right.\right.$. Since $\bigcap_{h>0} \mathcal{A}_{\alpha}(\lambda, h)=\mathrm{F}_{\alpha}(\lambda)$, we have shown that

$$
\overline{\operatorname{co}-\operatorname{dim}}\left(\mathrm{F}_{\alpha}(\lambda)\right) \leqslant \lambda^{2} .
$$

Unravelling the notation, this implies Eq. (1.5) (i.e., the lower bound in Theorem 1.1). It also implies the lower bound in Theorem 1.4.

\section{§5. THEOREM 1.3: UpPeR BOUnd}

For $\eta, R>1$, and $j \geqslant 1$, define,

$$
\mathcal{G}(j) \triangleq\left\{0 \leqslant m \leqslant R^{\eta j}:\left|W\left(m R^{-\eta j}\right)\right| \leqslant 2 \sqrt{\eta j R^{-\eta j} \ln R}\right\} .
$$

The notation is motivated by the following description: we think of $I_{m, j}^{\eta}$ (see $(3.2))$ as "good" if $m \in \mathcal{G}(j)$. Otherwise, $I_{m, j}^{\eta}$ is deemed "bad". We also recall Eq. (3.1) with $\alpha=1$ (thus replacing $X$ by $W$ in (3.1)). In analogy with the definition of $\mathcal{J}_{k}$ (see (3.4)), we define,

$$
\partial_{k}^{\prime} \triangleq \sum_{j \geqslant k} \sum_{m \in \mathcal{G}(j)} \mathbb{1}_{\left\{I_{m, j}^{\eta} \cap \mathrm{F}_{1}\left(\lambda, R^{-j}\right) \neq \varnothing\right\}}
$$

By the independence of the increments of $W,\left\{I_{m, j}^{\eta} \cap \mathrm{F}_{1}\left(\lambda, R^{-j}\right) \neq \varnothing\right\}$ is independent of $\{m \in \mathcal{G}(j)\}$. Recalling (3.3), we see that

$$
\left\|\mathcal{J}_{k}^{\prime}\right\|_{1}=\sum_{j \geqslant k} \sum_{m \geqslant 0} \mathbb{P}(m \in \mathcal{G}(j)) P_{m, j}^{\lambda, 1} .
$$


Since $|W(1)|$ has a probability density which is uniformly bounded above by 1 , for all $0 \leqslant m \leqslant R^{\eta j}$,

$$
\mathbb{P}(m \in \mathcal{G}(j)) \leqslant 4\left(\sqrt{\frac{\eta j \ln R}{m}} \wedge 1\right) .
$$

Next, fix $\varepsilon \in] 0,1\left[\right.$. By Lemma 3.1, there exists $J_{3} \triangleq J_{1}(\varepsilon, 1, \eta, \lambda, R) \in[2, \infty[$, such that for all $j \geqslant J_{3}$ and all $m \geqslant 0, P_{m, j}^{\lambda, 1} \leqslant R^{-\lambda^{2}(1-\varepsilon) j}$. Using (5.3), we see that for all $k \geqslant J_{3}$,

$$
\left\|\mathcal{J}_{k}^{\prime}\right\|_{1} \leqslant 4 \sum_{j \geqslant k} \sum_{m=0}^{\left[R^{\eta j}\right]}\left(\sqrt{\frac{\eta j \ln R}{m}} \wedge 1\right) R^{-\lambda^{2}(1-\varepsilon) j} .
$$

If we choose $\lambda^{2}(1-\varepsilon)>\eta / 2$, then a few lines of calculations reveal that $\sum_{k}\left\|\partial_{k}^{\prime}\right\|_{1}<\infty$. By the Borel-Cantelli lemma, there exists a finite random variable $k_{1}$, such that with probability one, for all $k \geqslant k_{1}, J_{k}^{\prime}=0$. In particular, with probability one, for all $j \geqslant k_{1}$,

$$
\mathbb{1}_{\{m \in \mathcal{G}(j)\}} \mathbb{1}_{\left\{I_{m, j}^{\eta} \cap \mathrm{F}_{1}\left(\lambda, R^{-j}\right) \neq \varnothing\right\}}=0 .
$$

By Lévy's modulus of continuity for $W$ (cf. (1.2)), there exists a finite random variable $k_{2}(\eta, R)$, such that with probability one, for all $j \geqslant k_{2}(\eta, R)$,

$$
\mathbb{1}_{\{m \in \mathcal{G}(j)\}} \geqslant \mathbb{1}_{\left\{I_{m, j}^{\eta} \cap z \neq \varnothing\right\}} .
$$

Eq. (5.4) shows that with probability one, for all $j \geqslant k_{3} \triangleq k_{1} \vee k_{2}(\eta, R)$, $\mathrm{F}_{1}\left(\lambda, R^{-j}\right) \cap z=\varnothing$. That is, almost surely, for all $j \geqslant k_{3}$,

$$
\sup _{t \in Z} \sup _{t \leqslant s \leqslant t+R^{-j}}|W(t+s)| \leqslant \lambda R^{-j / 2} \psi\left(R^{-j}\right) .
$$

If $h \leqslant R^{-k_{3}}$, there exists $j \geqslant k_{3}$, such that $R^{-j-1} \leqslant h \leqslant R^{-j}$. By monotonicity,

$$
\sup _{t \in z} \sup _{t \leqslant s \leqslant t+h}|W(t+s)| \leqslant \lambda R^{-j / 2} \psi\left(R^{-j}\right) \leqslant \lambda R^{1 / 2} h^{1 / 2} \psi(h) .
$$

In particular, we have shown that as long as $\lambda^{2}(1-\varepsilon)>\eta / 2$, then almost surely,

$$
\limsup _{h \rightarrow 0^{+}} \sup _{t \in z} \frac{|W(t+h)|}{h^{1 / 2} \psi(h)} \leqslant \lambda R^{1 / 2}
$$

Along rational sequences (and in this order), let $\eta, R \rightarrow 1^{+}, \varepsilon \rightarrow 0^{+}$and $\lambda^{2} \downarrow \frac{1}{2}$ to see that with probability one,

$$
\limsup _{h \rightarrow 0^{+}} \sup _{t \in \mathcal{Z}} \frac{|W(t+h)|}{h^{1 / 2} \psi(h)} \leqslant \frac{1}{\sqrt{2}} .
$$




\section{§6. TheOrem 1.4: UpPer Bound}

Recall Eqs. (1.6) and (3.1). We begin with a "regularization scheme" which is used later in our good covering for dimension calculations.

Lemma 6.1. For all integers $k \geqslant 1$ and all reals $\theta \in] 0,1[$ and $R>1$,

$$
\mathrm{F}_{\alpha}(\lambda) \subset \bigcup_{j \geqslant k} \mathrm{~F}_{\alpha}\left(\theta \lambda R^{-\alpha / 2}, R^{-j+1}\right)
$$

Proof. From first principles, it follows that for all $\beta<\lambda, \mathrm{F}_{\alpha}(\lambda) \subset$ $\bigcup_{h>0} \bigcup_{0<\delta<h} \mathrm{~F}_{\alpha}(\beta, \delta)$. Let $\delta<h<1$, say $R^{-j} \leqslant \delta \leqslant R^{-j+1}$. For all $t \in F_{\alpha}(\beta, \delta)$,

$\sup _{t \leqslant s \leqslant t+R^{-j+1}}|X(s)-X(t)| \geqslant \beta R^{-j \alpha / 2} \psi\left(R^{-j+1}\right)=\beta R^{-\alpha / 2} R^{-(j-1) \alpha / 2} \psi\left(R^{-j+1}\right)$.

We have used the monotonicity properties of $\psi$. This implies that $t \in$ $F_{\alpha}\left(\beta R^{-\alpha / 2}, R^{-(j-1)}\right)$. The result follows.

We are prepared to demonstrate the upper bound in Theorem 1.4.

Proof of Theorem 1.4: upper bound. Fix $R, \eta>1, \theta \in] 0,1\left[\right.$ and recall $I_{m, j}^{\eta}$ from (3.2). From Lemma 6.1, it is apparent that for any integer $k \geqslant 1$, we have the following covering of $\mathrm{F}_{\alpha}(\lambda)$ :

$$
\mathrm{F}_{\alpha}(\lambda) \subset \bigcup_{j=k}^{\infty} \bigcup_{m=1}^{\infty} I_{m, j}^{\eta} \cap \mathrm{F}_{\alpha}\left(\theta \lambda R^{-\alpha / 2}, R^{-j+1}\right) .
$$

By (3.3) and Lemma 3.1, for all $\varepsilon>0$, there exists $J_{4}=J_{4}(\varepsilon, \alpha, \eta, \lambda, R, \theta) \in$ $\left[2, \infty\left[\right.\right.$, such that for all $j \geqslant J_{4}$,

$$
\mathbb{P}\left(I_{m, j}^{\eta} \cap \mathrm{F}_{\alpha}\left(\theta \lambda R^{-\alpha / 2}, R^{-j+1}\right) \neq \varnothing\right) \leqslant R^{-(1-\varepsilon) \theta^{2} \lambda^{2} R^{-2 \alpha} j} .
$$

For any $s \geqslant 0$ and every integer $k \geqslant 1$, define,

$$
\mathcal{J}_{k}(s) \triangleq \sum_{j \geqslant k} \sum_{m \leqslant R^{\eta j}+1}\left|I_{m, j}^{\eta}\right|^{s} \mathbb{1}_{\left\{I_{m, j}^{\eta} \cap E \neq \varnothing\right\}} \mathbb{1}_{\left\{I_{m, j}^{\eta} \cap \mathrm{F}_{\alpha}\left(\theta \lambda R^{-\alpha / 2}, R^{-j+1}\right) \neq \varnothing\right\}} .
$$

By (6.2), for all $k \geqslant J_{4}$,

$$
\left\|\mathcal{J}_{k}(s)\right\|_{1} \leqslant \sum_{j \geqslant k} M\left(\left(1+R^{\eta j}\right)^{-1} ; E\right) R^{-\eta s j} R^{-(1-\varepsilon) \theta^{2} \lambda^{2} R^{-2 \alpha} j} .
$$


(Recall from $\S 3$ that $M(\varepsilon ; E)$ is the $\varepsilon$-capacity of $E$.) Note that if we enlarge $J_{4}$ further, then for all $\beta>\overline{\operatorname{dim}}_{M}(E)$ and all $j \geqslant J_{4}$, we can also ensure that $M\left(\left(1+R^{\eta j}\right)^{-1} ; E\right) \leqslant R^{\eta \beta j}$. Suppose $\beta>\overline{\operatorname{dim}}_{M}(E)$ and

$$
\eta s>\eta \beta-(1-\varepsilon) \theta^{2} \lambda^{2} R^{-2 \alpha} .
$$

It follows that $\sum_{k}\left\|\partial_{k}(s)\right\|_{1}<\infty$. By the Borel-Cantelli lemma, $\lim _{k \rightarrow \infty} \mathcal{J}_{k}(s)=$ 0, a.s. From (6.1) and the definition of Hausdorff dimension, it follows that for any $s$ satisfying (6.3),

$$
\Lambda^{s}\left(E \cap \mathrm{F}_{\alpha}(\lambda)\right) \leqslant \lim _{k \rightarrow \infty} \partial_{k}(s)=0, \quad \text { a.s. }
$$

Therefore, almost surely,

$$
\operatorname{dim}\left(E \cap \mathrm{F}_{\alpha}(\lambda)\right) \leqslant s .
$$

Letting $\beta \downarrow \overline{\operatorname{dim}}_{M}(E), \varepsilon \downarrow 0, \eta, R \downarrow 1, \theta \uparrow 1$ in this order and along rational sequences, we obtain

$$
\operatorname{dim}\left(E \cap \mathrm{F}_{\alpha}(\lambda)\right) \leqslant \overline{\operatorname{dim}}_{M}(E)-\lambda^{2}, \quad \text { a.s. }
$$

By $\left[17\right.$, pp. 57 and 81], for every $G \in \mathbf{S}_{0}^{1}$,

$$
\begin{aligned}
\operatorname{dim}(G) & =\inf _{G=\cup_{i=1}^{\infty} G_{i}} \sup _{i} \operatorname{dim}\left(G_{i}\right), \\
\operatorname{dim}_{\mathrm{P}}(G) & =\inf _{G=\cup_{i=1}^{\infty} G_{i}} \sup _{i} \overline{\operatorname{dim}}_{M}\left(G_{i}\right),
\end{aligned}
$$

where the $G_{i}$ 's are assumed to be bounded. Thus,

$$
\operatorname{dim}\left(E \cap \mathrm{F}_{\alpha}(\lambda)\right) \leqslant \operatorname{dim}_{\mathrm{P}}(E)-\lambda^{2}, \quad \text { a.s., }
$$

which is the desired upper bound.

\section{§7. Proof of Theorem 1.5: Upper Bound}

Recall the notation of $\S 3$ and $\S 6$, and $\mathcal{G}(j)$ from (5.1). By Lemma 6.1 and (5.5), for any $\eta, R>1$ and $\theta \in] 0,1[$, there exists a finite random variable $K$ such that almost surely for all $k \geqslant K$,

$$
Z \cap \mathrm{F}_{1}(\lambda) \subset \bigcup_{j \geqslant k} \bigcup_{m \in \mathcal{G}(j)} I_{m, j}^{\eta} \cap \mathrm{F}_{1}\left(\theta \lambda R^{-1 / 2}, R^{-j+1}\right) .
$$

Next, we show that the above is a fairly economical covering. Since $W$ has independent increments, for any $s>0$,

$$
\begin{aligned}
& \sum_{j \geqslant k} \sum_{0 \leqslant R^{\eta j}+1}\left|I_{m, j}^{\eta}\right|^{s} \mathbb{P}\left(I_{m, j}^{\eta} \cap \mathrm{F}_{1}\left(\theta \lambda R^{-1 / 2}, R^{-j+1}\right) \neq \varnothing, m \in \mathcal{G}(j)\right) \\
& =\sum_{j \geqslant k} \sum_{0 \leqslant m<R^{\eta j}+1}\left|I_{m, j}^{\eta}\right|^{s} \mathbb{P}\left(I_{m, j}^{\eta} \cap \mathrm{F}_{1}\left(\theta \lambda R^{-1 / 2}, R^{-j+1}\right) \neq \varnothing\right) \mathbb{P}(m \in \mathcal{G}(j)) .
\end{aligned}
$$


By (5.3),

$$
\begin{aligned}
& \sum_{j \geqslant k} \sum_{0 \leqslant m<R^{\eta j}+1}\left|I_{m, j}^{\eta}\right|^{s} \mathbb{P}\left(I_{m, j}^{\eta} \cap \mathrm{F}_{1}\left(\theta \lambda R^{-1 / 2}, R^{-j+1}\right) \neq \varnothing, m \in \mathcal{G}(j)\right) \\
& \leqslant 4 \sum_{j \geqslant k} \sum_{m=0}^{\left[R^{\eta j}\right]+1}\left|I_{m, j}^{\eta}\right|^{s} \mathbb{P}\left(I_{m, j}^{\eta} \cap \mathrm{F}_{1}\left(\theta \lambda R^{-1 / 2}, R^{-j+1}\right) \neq \varnothing\right)\left(\sqrt{\frac{\eta j \ln R}{m}} \wedge 1\right) .
\end{aligned}
$$

Using Lemma 3.1, we see that for all $\varepsilon \in] 0,1\left[\right.$, there exists $J_{5}=J_{5}(\varepsilon, \theta, \eta, \lambda, R) \in$ $\left[2, \infty\left[\right.\right.$, such that for all $k \geqslant J_{5}$,

$$
\begin{array}{r}
\sum_{j \geqslant k} \sum_{0 \leqslant m<R^{\eta j}+1}\left|I_{m, j}^{\eta}\right|^{s} \mathbb{P}\left(I_{m, j}^{\eta} \cap \mathrm{F}_{1}\left(\theta \lambda R^{-1 / 2}, R^{-j+1}\right) \neq \varnothing, m \in \mathcal{G}(j)\right) \\
\leqslant 4 \sum_{j \geqslant k} R^{-\eta s j} R^{-(1-\varepsilon) \theta^{2} \lambda^{2} R^{-1} j} \sum_{m=0}^{\left[R^{\eta j}\right]+1}\left(\sqrt{\frac{\eta j \ln R}{m}} \wedge 1\right) .
\end{array}
$$

In particular, if

$$
\eta s>\frac{\eta}{2}-(1-\varepsilon) \theta^{2} \lambda^{2} R^{-1}
$$

then,

$$
\lim _{k \rightarrow \infty} \sum_{j \geqslant k} \sum_{m \in \mathcal{G}(j)}\left|I_{m, j}^{\eta}\right|^{s} \mathbb{1}_{\left\{I_{m, j}^{\eta} \cap F_{1}\left(\theta \lambda R^{-1 / 2}, R^{-j+1}\right) \neq \varnothing\right\}}=0, \quad \text { a.s. }
$$

Thanks to (7.1), we can deduce that for any $s$ satisfying $(7.2), \Lambda^{s}\left(Z \cap F_{1}(\lambda)\right)=0$, almost surely. In particular, almost surely, $\operatorname{dim}\left(z \cap \mathrm{F}_{1}(\lambda)\right) \leqslant s$. Let $\varepsilon \downarrow 0, \theta \uparrow 1$ and $R, \eta \downarrow 1$ in this order to see that with probability one, $\operatorname{dim}\left(Z \cap \mathrm{F}_{1}(\lambda)\right) \leqslant \frac{1}{2}-$ $\lambda^{2}$. This is the desired upper bound.

\section{§8. Proofs of Theorems 1.3 AND 1.5: LOWER BOUNDS}

The main result of this section is the following which may be of independent interest.

Theorem 8.1. Fix a compact set $E \subset[0,1]$. Then,

$$
\operatorname{dim}(E)>\lambda^{2}+\frac{1}{2} \Longrightarrow \mathbb{P}\left(Z \cap \mathrm{F}_{1}(\lambda) \cap E \neq \varnothing\right)=1 .
$$

As the lower bounds in Theorems 1.3 and 1.5 follow immediately from the above, the rest of this section is devoted to proving Theorem 8.1.

Suppose $E \subset[0,1]$ is compact, $\mu \in \mathcal{P}_{+}(E)$ and $h, \lambda>0$. Define, 
SÉMINAIRE DE PROBABILITÉS XXXIV, Lec. Notes in Math. 393-416 (2000)

$$
J_{\mu}(h ; \lambda) \triangleq \int_{0}^{1} \mu(d s) \mathbb{1}_{\{|W(s)|<h\}} \mathbb{1}_{\left\{W(s+h)-W(s)>\lambda h^{1 / 2} \psi(h)\right\}} .
$$

For all $\mu \in \mathcal{P}_{+}(E)$ and any $h, \beta>0$, define the following:

$$
\begin{aligned}
& S_{h}(\mu) \triangleq \sup _{0 \leqslant s \leqslant h} \int_{s}^{h} \frac{\mu(d t)}{\sqrt{t-s}}, \\
& \widetilde{S}_{h}(\mu) \triangleq \sup _{0 \leqslant s \leqslant 1} \int_{s}^{(s+h) \wedge 1} \frac{\mu(d t)}{\sqrt{t-s}} .
\end{aligned}
$$

The proof of Theorem 8.1 is divided into several steps. In analytical terms, our first result is an estimate, uniform in $s \in[0, h]$, for the $1 / 2-$ potential of a measure $\mu$ restricted to an interval $[s, h]$. Indeed, recalling $A_{\beta}(\mu)$ from $(2.1)$, we have the following:

Lemma 8.2. Suppose $\mu \in \mathcal{P}_{+}(E)$. Then for all $\beta>1 / 2$ and all $h>0$,

$$
\begin{aligned}
& S_{h}(\mu) \leqslant \frac{2 e^{\beta}}{2 \beta-1} A_{\beta}(\mu) h^{\beta-\frac{1}{2}}, \\
& \widetilde{S}_{h}(\mu) \leqslant \frac{2 e^{\beta}}{2 \beta-1} A_{\beta}(\mu) h^{\beta-\frac{1}{2}} .
\end{aligned}
$$

Proof. Without loss of generality, we can assume that $A_{\beta}(\mu)<\infty$ for some $\beta>1 / 2$ (otherwise, there is nothing to prove). We proceed with an approximate integration by parts: for all $0 \leqslant s \leqslant h$,

$$
\begin{aligned}
& \int_{s}^{h} \mu(d t)(t-s)^{-1 / 2}=\sum_{j=0}^{\infty} \int_{s+(h-s) e^{-j-1}}^{s+(h-s) e^{-j}} \mu(d t)(t-s)^{-1 / 2} \\
& \leqslant(h-s)^{-1 / 2} \sum_{j=0}^{\infty} e^{(j+1) / 2} \mu\left[s+(h-s) e^{-j-1}, s+(h-s) e^{-j}\right] \\
& \leqslant e^{1 / 2}(h-s)^{\beta-1 / 2} A_{\beta}(\mu) \sum_{j=0}^{\infty} \exp \left(-j\left(\beta-\frac{1}{2}\right)\right),
\end{aligned}
$$

which yields (8.1). The estimate (8.2) can be checked in the same way.

Our next two lemmas are moment estimates for $J_{\mu}$.

Lemma 8.3. Suppose $\mu \in \mathcal{P}_{+}(E)$ is fixed. For every $\varepsilon>0$, there exists an $h_{\varepsilon}>0$, such that for all $\left.h \in\right] 0, h_{\varepsilon}[$, and all $\lambda>0$,

$$
\left\|J_{\mu}(h ; \lambda)\right\|_{1} \geqslant \sqrt{\frac{2}{\pi e}}(1-\varepsilon) h \bar{\Phi}(\lambda \psi(h)) .
$$


SÉMINAIRE DE PROBABILITÉS XXXIV, Lec. Notes in Math. 393-416 (2000)

Proof. By the independence of the increments of $W$,

$$
\left\|J_{\mu}(h ; \lambda)\right\|_{1}=\bar{\Phi}(\lambda \psi(h)) \int_{0}^{1} \mu(d s) \mathbb{P}(|W(s)| \leqslant h) .
$$

A direct calculation reveals that if $r \in] 0,1[, \mathbb{P}(|W(1)| \leqslant r) \geqslant \sqrt{2 /(\pi e)} r$. Therefore, by Brownian scaling, for every $h \in] 0,1[$,

$$
\begin{aligned}
\left\|J_{\mu}(h ; \lambda)\right\|_{1} & \geqslant \sqrt{\frac{2}{\pi e}} h \bar{\Phi}(\lambda \psi(h)) \int_{h^{2}}^{1} \frac{\mu(d s)}{\sqrt{s}} \\
& \geqslant \sqrt{\frac{2}{\pi e}} \mu\left[h^{2}, 1\right] h \bar{\Phi}(\lambda \psi(h)) .
\end{aligned}
$$

The lemma follows upon taking $h>0$ small enough.

Lemma 8.4. Fix $\mu \in \mathcal{P}_{+}(E)$. Suppose $A_{\beta}(\mu)<\infty$ for some $\beta>1 / 2$. Then, for all $h, \lambda>0$,

$$
\left\|J_{\mu}(h ; \lambda)\right\|_{2}^{2} \leqslant \frac{8 e^{2 \beta}}{(2 \beta-1)^{2}} A_{\beta}^{2}(\mu)\left\{2 h^{\beta+3 / 2} \bar{\Phi}(\lambda \psi(h))+h^{2} \bar{\Phi}^{2}(\lambda \psi(h))\right\} .
$$

Proof. To save space, for all $h, t \geqslant 0$, define,

$$
\Delta_{h} W(t) \triangleq W(t+h)-W(t) .
$$

By the independence of the increments of $W$,

$$
\begin{aligned}
& \left\|J_{\mu}(h ; \lambda)\right\|_{2}^{2}=2 \| \int_{0}^{1} \mu(d t) \mathbb{1}_{\{|W(t)| \leqslant h\}} \mathbb{1}_{\left\{\Delta_{h} W(t) \geqslant \lambda h^{1 / 2} \psi(h)\right\}} \times \\
& \quad \times \int_{0}^{t} \mu(d s) \mathbb{1}_{\{|W(s)| \leqslant h\}} \mathbb{1}_{\left\{\Delta_{h} W(s) \geqslant \lambda h^{1 / 2} \psi(h)\right\}} \|_{1} \\
& =2 \bar{\Phi}(\lambda \psi(h))\left\|\int_{0}^{1} \mu(d t) \mathbb{1}_{\{|W(t)| \leqslant h\}} \int_{0}^{t} \mu(d s) \mathbb{1}_{\{|W(s)| \leqslant h\}} \mathbb{1}_{\left\{\Delta_{h} W(s) \geqslant \lambda h^{1 / 2} \psi(h)\right\}}\right\|_{1} \\
& \leqslant 2 \bar{\Phi}(\lambda \psi(h))\left[T_{1}+T_{2}\right]
\end{aligned}
$$

where,

$$
\begin{aligned}
& T_{1} \triangleq\left\|\int_{h}^{1} \mu(d t) \mathbb{1}_{\{|W(t)| \leqslant h\}} \int_{0}^{(t-h)^{+}} \mu(d s) \mathbb{1}_{\{|W(s)| \leqslant h\}} \mathbb{1}_{\left\{\Delta_{h} W(s) \geqslant \lambda h^{1 / 2} \psi(h)\right\}}\right\|_{1}, \\
& T_{2} \triangleq\left\|\int_{0}^{1} \mu(d t) \mathbb{1}_{\{|W(t)| \leqslant h\}} \int_{(t-h)^{+}}^{t} \mu(d s) \mathbb{1}_{\{|W(s)| \leqslant h\}}\right\|_{1} .
\end{aligned}
$$

We will estimate $T_{1}$ and $T_{2}$ in turn. 
We will need the following consequence of Gaussian laws: for all $s, h>0$,

$$
\mathbb{P}(|W(s)| \leqslant h) \leqslant s^{-1 / 2} h .
$$

First, we estimate $T_{1}$. Note that,

$$
T_{1}=\int_{h}^{1} \mu(d t) \int_{0}^{t-h} \mu(d s) \mathbb{P}\left(|W(t)| \leqslant h,|W(s)| \leqslant h, \Delta_{h} W(s) \geqslant \lambda h^{1 / 2} \psi(h)\right) .
$$

Suppose $t \in[h, 1]$ and $s \in[0, t-h]$. Then $s \leqslant s+h \leqslant t$, and we have,

$$
\begin{aligned}
\mathbb{P}(|W(t)| \leqslant h & \mid W(r) ; r \leqslant s+h) \\
& =\mathbb{P}(|W(t)-W(s+h)+W(s+h)| \leqslant h \mid W(r) ; r \leqslant s+h) \\
& \leqslant \sup _{\zeta \in \mathbb{R}} \mathbb{P}(|W(t-s-h)+\zeta| \leqslant h) .
\end{aligned}
$$

On the other hand, $W(t-s-h)$ has a Gaussian distribution. By unimodality of the latter,

$$
\mathbb{P}(|W(t)| \leqslant h \mid W(r) ; r \leqslant s+h) \leqslant \mathbb{P}(|W(t-s-h)| \leqslant h) .
$$

(This actually is a particular case of T.W. Anderson's inequality for general Gaussian shifted balls). Using (8.5) and the principle of conditioning,

$$
\begin{aligned}
T_{1} & \leqslant \int_{h}^{1} \mu(d t) \int_{0}^{t-h} \mu(d s) \mathbb{P}(|W(t-s-h)| \leqslant h) \times \\
& \times \mathbb{P}\left(|W(s)| \leqslant h, \Delta_{h} W(s) \geqslant \lambda h^{1 / 2} \psi(h)\right) \\
& \leqslant \bar{\Phi}(\lambda \psi(h)) \int_{h}^{1} \mu(d t) \int_{0}^{t-h} \mu(d s) \mathbb{P}(|W(t-s-h)| \leqslant h) \mathbb{P}(|W(s)| \leqslant h) .
\end{aligned}
$$

By (8.4),

$$
T_{1} \leqslant h^{2} \bar{\Phi}(\lambda \psi(h)) \int_{h}^{1} \mu(d t) \int_{0}^{t-h} \mu(d s) \frac{1}{\sqrt{s(t-s-h)}} .
$$

Changing the order of integration, we arrive at the following estimate:

$$
\begin{aligned}
T_{1} & \leqslant h^{2} \bar{\Phi}(\lambda \psi(h)) \int_{0}^{1-h} \frac{\mu(d s)}{\sqrt{s}} \int_{s+h}^{1} \frac{\mu(d t)}{\sqrt{t-s-h}} \\
& \leqslant h^{2} \bar{\Phi}(\lambda \psi(h)) S_{1}^{2}(\mu) \\
& \leqslant \frac{4 e^{2 \beta}}{(2 \beta-1)^{2}} A_{\beta}^{2}(\mu) h^{2} \bar{\Phi}(\lambda \psi(h)),
\end{aligned}
$$

by Lemma 8.2 . Next, we estimate $T_{2}$. By another unimodality argument, for all $t \geqslant s$ and all $h>0$,

$$
\mathbb{P}(|W(s)| \leqslant h,|W(t)| \leqslant h) \leqslant \mathbb{P}(|W(s)| \leqslant h) \mathbb{P}(|W(t-s)| \leqslant h) .
$$


Applying (8.4), $\mathbb{P}(|W(s)| \leqslant h,|W(t)| \leqslant h) \leqslant h^{2} / \sqrt{s(t-s)}$. Therefore,

$$
\begin{aligned}
T_{2} & \leqslant h^{2} \int_{0}^{1} \mu(d t) \int_{(t-h)^{+}}^{t} \mu(d s) \frac{1}{\sqrt{s(t-s)}} \\
& =h^{2}\left(T_{2,1}+T_{2,2}\right),
\end{aligned}
$$

where,

$$
\begin{aligned}
& T_{2,1} \triangleq \int_{0}^{h} \mu(d t) \int_{0}^{t} \mu(d s) \frac{1}{\sqrt{s(t-s)}} \\
& T_{2,2} \triangleq \int_{h}^{1} \mu(d t) \int_{t-h}^{t} \mu(d s) \frac{1}{\sqrt{s(t-s)}} .
\end{aligned}
$$

To estimate $T_{2,1}$, reverse the order of integration:

$$
\begin{aligned}
T_{2,1} & =\int_{0}^{h} \frac{\mu(d s)}{\sqrt{s}} \int_{s}^{h} \frac{\mu(d t)}{\sqrt{t-s}} \leqslant S_{h}^{2}(\mu) \\
& \leqslant \frac{4 e^{2 \beta}}{(2 \beta-1)^{2}} A_{\beta}^{2}(\mu) h^{2 \beta-1},
\end{aligned}
$$

by Lemma 8.2. Similarly,

$$
\begin{aligned}
T_{2,2} & =\int_{0}^{1} \frac{\mu(d s)}{\sqrt{s}} \int_{s}^{(s+h) \wedge 1} \frac{\mu(d t)}{\sqrt{t-s}} \\
& \leqslant S_{1}(\mu) \widetilde{S}_{h}(\mu) \\
& \leqslant \frac{4 e^{2 \beta}}{(2 \beta-1)^{2}} A_{\beta}^{2}(\mu) h^{\beta-1 / 2} .
\end{aligned}
$$

Since $h<1$ and $\beta>1 / 2$, using the above, (8.8) and (8.7), we arrive at the following:

$$
T_{2} \leqslant \frac{8 e^{2 \beta}}{(2 \beta-1)^{2}} A_{\beta}^{2}(\mu) h^{\beta+3 / 2} .
$$

Use this, together with (8.6) and (8.3) in this order to get the result.

We are ready for the main result of this section:

Proof of Theorem 8.1. For $\lambda, h>0$, recall (4.9) and define,

$$
Z(h) \triangleq\{s \in[0,1]:|W(s)|<h\} .
$$

Path continuity of $W$ alone implies that $Z(h) \cap \mathcal{A}_{1}(\lambda, h)$ is an open random set. We estimate the probability that it intersects $E$. By Frostman's lemma, for all $\beta<\operatorname{dim}(E)$, there exists $\mu \in \mathcal{P}_{+}(E)$ such that $A_{\beta}(\mu)<\infty$. Let us fix a $\mu$ corresponding to an arbitrary but fixed choice of $\beta$ satisfying:

$$
\lambda^{2}+\frac{1}{2}<\beta<\operatorname{dim}(E) .
$$


Applying Lemmas 8.3 and 8.4 to this choice of $\mu$, we see that for all $\varepsilon>0$, there exists $h_{\varepsilon}>0$, such that for all $\left.h \in\right] 0, h_{\varepsilon}[$,

$$
\frac{\left\|J_{\mu}(h ; \lambda)\right\|_{1}^{2}}{\left\|J_{\mu}(h ; \lambda)\right\|_{2}^{2}} \geqslant \gamma_{\varepsilon, \beta}\left[\frac{2 h^{\beta-1 / 2}}{\bar{\Phi}(\lambda \psi(h))}+1\right]^{-1},
$$

where,

$$
\gamma_{\varepsilon, \beta} \triangleq \frac{(2 \beta-1)^{2}(1-\varepsilon)^{2}}{4 \pi e^{1+2 \beta} A_{\beta}^{2}(\mu)} .
$$

According to Mill's ratio for Gaussian tails (see (4.4)), we can pick $h_{\lambda}$ so small that for each and every $h \in] 0, h_{\lambda}\left[, \bar{\Phi}(\lambda \psi(h)) \geqslant h^{\lambda^{2}} / 4 \lambda \sqrt{\ln (1 / h)}\right.$. Therefore, for all $h \in] 0, h_{\varepsilon} \wedge h_{\lambda}[$,

$$
\frac{\left\|J_{\mu}(h ; \lambda)\right\|_{1}^{2}}{\left\|J_{\mu}(h ; \lambda)\right\|_{2}^{2}} \geqslant \gamma_{\varepsilon, \beta}\left[8 \lambda h^{\beta-\lambda^{2}-1 / 2} \sqrt{\ln (1 / h)}+1\right]^{-1} .
$$

By (8.9), $\liminf _{h \rightarrow 0^{+}}\left\|J_{\mu}(h ; \lambda)\right\|_{1}^{2}\left\|J_{\mu}(h ; \lambda)\right\|_{2}^{-2} \geqslant \gamma_{\varepsilon, \beta}>0$. By the PaleyZygmund inequality $([8, \mathrm{p} .8])$,

$$
\liminf _{h \rightarrow 0^{+}} \mathbb{P}\left(J_{\mu}(h ; \lambda)>0\right) \geqslant \gamma_{\varepsilon, \beta}>0 .
$$

Note that the event $\left(J_{\mu}(h ; \lambda)>0\right)$ implies that $Z(h) \cap \mathcal{A}_{1}(\lambda, h)$ intersects $E$. Hence,

$$
\liminf _{h \rightarrow 0^{+}} \mathbb{P}\left(Z(h) \cap \mathcal{A}_{1}(\lambda, h) \cap E \neq \varnothing\right) \geqslant \gamma_{\varepsilon, \beta}>0 .
$$

However, as $h \downarrow 0$, the (random) open set $Z(h) \cap \mathcal{A}_{1}(\lambda, h)$ decreases to $Z \cap F_{1}(\lambda)$. Adapting Theorem 2.5 to the positive probability case, we can conclude that $\mathbb{P}\left(Z \cap F_{1}(\lambda) \cap E \neq \varnothing\right) \geqslant \gamma_{\varepsilon, \beta}>0$. Note that the only requirement on $E$ was that $\operatorname{dim}(E)>\lambda^{2}+1 / 2$. Since Hausdorff dimension is scale invariant, we see that for any real number $s \in] 0,1[$,

$$
\mathbb{P}\left(Z \cap \mathrm{F}_{1}(\lambda) \cap s^{-1} E \neq \varnothing\right) \geqslant \gamma_{\varepsilon, \beta},
$$

where $s^{-1} E \triangleq\{r / s: r \in E\}$. We finish the proof by showing that this probability is actually 1 . Fix $s \in] 0,1$ [ and observe from Brownian scaling that $Z \cap F_{1}(\lambda)$ has the same distribution as $Z \cap \mathrm{F}_{1}(\lambda) \cap[0, s]$ in the sense that for all $G \in \mathbf{S}_{0}^{1}$,

$$
\mathbb{P}\left(Z \cap \mathrm{F}_{1}(\lambda) \cap G \neq \varnothing\right)=\mathbb{P}\left(Z \cap \mathrm{F}_{1}(\lambda) \cap[0, s] \cap s G \neq \varnothing\right) .
$$

In particular, for all $s \in] 0,1\left[, \mathbb{P}\left(Z \cap \mathrm{F}_{1}(\lambda) \cap[0, s] \cap E \neq \varnothing\right) \geqslant \gamma_{\varepsilon, \beta}>0\right.$, Note that $z \cap \mathrm{F}_{1}(\lambda) \cap E \cap[0, s]$ is increasing in $s$. Thus,

$$
\mathbb{P}\left(\bigcap_{s \in] 0,1[}\left\{z \cap \mathrm{F}_{1}(\lambda) \cap E \cap[0, s] \neq \varnothing\right\}\right) \geqslant \gamma_{\varepsilon, \beta} .
$$

Let $C \triangleq \cap_{s \in] 0,1[}\left(Z \cap \mathrm{F}_{1}(\lambda) \cap E \cap[0, s] \neq \varnothing\right)$. Observe that $C$ is measurable with respect to the germ field of $W$ at 0 and we have just argued that $\mathbb{P}(C) \geqslant \gamma_{\varepsilon, \beta}>0$. By Blumenthal's 0-1 law, $\mathbb{P}(C)=1$. Since $\left(Z \cap \mathrm{F}_{1}(\lambda) \cap E \neq \varnothing\right) \supset C$, the result follows for $\mathrm{F}_{1}$. 


\section{REFERENCES}

[1] M.T. Barlow And E. Perkins (1984). Levels at which every Brownian excursion is exceptional. Sém. Prob. XVIII, Lecture Notes in Math. 1059, 1-28, Springer-Verlag, New York.

[2] M. Csörgő And P. RÉvÉsz (1981). Strong Approximations in Probability and Statistics, Academic Press, New York.

[3] P. Deheuvels and M.A. Lifshits (1997). On the Hausdorff dimension of the set generated by exceptional oscillations of a Wiener process. Studia Sci. Math. Hung., 33, 75-110.

[4] P. Deheuvels and D.M. Mason (1997). Random fractal functional laws of the iterated logarithm. (preprint)

[5] R.M. Dudley (1984). A Course on Empirical Processes. École d'Été de St. Flour 1982. Lecture Notes in Mathematics 1097. Springer, Berlin.

[6] J. Hawkes (1971). On the Hausdorff dimension of the range of a stable process with a Borel set. Z. Wahr. verw. Geb., 19, 90-102.

[7] J. Hawkes (1981). Trees generated by a simple branching process. J. London Math. Soc., 24, 373-384.

[8] J.-P. Kahane (1985). Some Random Series of Functions, second edition. Cambridge University Press, Cambridge.

[9] R. Kaufman (1974). Large increments of Brownian Motion. Nagoya Math. J., 56, 139-145.

[10] D. Khoshnevisan, Y. Peres and Y. Xiao (1998). Limsup random fractals. In preparation.

[11] N. KôNo (1977). The exact Hausdorff measure of irregularity points for a Brownian path. Z. Wahr. verw. Geb., 40, 257-282.

[12] M. Ledoux and M. Talagrand (1991). Probability in Banach Space, Isoperimetry and Processes, Springer-Verlag, Heidelberg-New York.

[13] P. LÉvy (1937). Théorie de l'Addition des Variables Aléatoires. GauthierVillars, Paris.

[14] R. Lyons (1980). Random walks and percolation on trees. Ann. Prob., 18, 931-958.

[15] M.B. Marcus (1968). Hölder conditions for Gaussian processes with stationary increments. Trans. Amer. Math. Soc., 134, 29-52.

[16] M.B. Marcus and J. Rosen (1992). Moduli of continuity of local times of strongly symmetric Markov processes via Gaussian processes. J. Theoretical Prob., 5, 791-825. 
SÉMINAIRE DE PROBABILITÉS XXXIV, Lec. Notes in Math. 393-416 (2000)

[17] P. Matilla (1995). Geometry of Sets and Measures in Euclidean Spaces, Fractals and Rectifiability, Cambridge University Press, Cambridge.

[18] S. Orey and S.J. TAYlor (1974). How often on a Brownian path does the law of the iterated logarithm fail? Proc. London Math. Soc., 28, 174-192.

[19] Y. Peres (1996). Remarks on intersection-equivalence and capacityequivalence. Ann. Inst. Henri Poincaré: Physique Théorique, 64, 339-347.

[20] E. Perkins and S.J. Taylor (1988), Measuring close approaches on a Brownian path, Ann. Prob., 16, 1458-1480.

[21] D. Revuz And M. Yor (1994). Continuous Martingales and Brownian Motion, second edition. Springer, Berlin.

[22] G.R. Shorack and J.A. Wellner (1986). Empirical Processes with Applications to Statistics. Wiley, New York.

[23] S.J. TAYLOR (1966). Multiple points for the sample paths of the symmetric stable process, Z. Wahr. ver. Geb., 5, 247-64.

[24] S.J. TAYLOR (1986). The measure theory of random fractals. Math. Proc. Camb. Phil. Soc., 100, 383-406.

Davar Khoshnevisan

Department of Mathematics

University of Utah

Salt Lake City, UT. 84112

U.S.A.

E-mail: davar@math.utah.edu
Zhan Shi

Laboratoire de Probabilités

Université Paris VI

4, Place Jussieu

75252 Paris Cedex 05, France

E-mail: shi@ccr.jussieu.fr 